to 10 years $(64.0-87.0 \%)$. The correlation coefficients varied from 0.1 to $0.4(\mathrm{p}<0.05)$.

Conclusions Although most of the correlations between the instruments were moderate, frailty prevalence varied largely according to the instrument used to assess the condition. These differences are justified because of the different domains assessed by each instrument.

\section{SP1-53 THE EFFECT OF SOCIAL DEPRIVATION ON WEIGHT IN THE UK CYSTIC FIBROSIS POPULATION}

doi:10.1136/jech.2011.142976n.30

${ }^{1} \mathrm{D}$ Taylor-Robinson, ${ }^{*} \mathrm{M}$ Whitehead, ${ }^{2} \mathrm{P}$ Diggle, ${ }^{1} \mathrm{R}$ Smyth. 'University of Liverpool, Liverpool, UK; ${ }^{2}$ Lancaster University, Lancaster, UK

Background Maintaining nutritional status is a key component of care in people with cystic fibrosis. Low socioeconomic status has been linked with poor outcomes in CF. We explored, for the first time in a UK-wide cohort, longitudinal weight gain and its relationship with socioeconomic status (SES).

Methods We undertook a retrospective longitudinal cohort study of 4346 people with cystic fibrosis aged $<20$ years $(21132$ observations) in UK CF registry between 1995 and 2006. Census based indices of multiple deprivation (IMD) from the UK constituent counties were used as small area measures of SES. Piecewise mixed model regression was used to estimate the effect of SES on weight-for-age z-score (WFA).

Results WFA was significantly lower in the most deprived quintile at all time points. The estimated WFA at birth (intercept) was -0.64 in the least deprived quintile compared to -1.31 in the most deprived (mean difference $0.6795 \%$ CI 0.42 to 0.92 ). The population WFA increased up to age three by 0.2 per year, and then declined subsequently by -0.033 per year. There was a significantly steeper improvement in WFA in the most deprived quintile in the first 3 years (mean difference per year $0.1395 \%$ CI 0.06 to 0.20 ), with no difference in the rate of decline subsequently.

Conclusions Social deprivation is associated with lower WFA in the UK cystic fibrosis population, but there is a period of increased weight gain in the first 3 years, highlighting the importance of early diagnosis and treatment.

\section{SP1-54 RESULTS OF A 3-YEAR WORKPLACE WELLNESS PROGRAM AMONG A WORK COHORT IN KUALA LUMPUR, MALAYSIA}

doi:10.1136/jech.2011.142976n.31

${ }^{1,2} \mathrm{~F}$ M Moy, ${ }^{* 1}$ A M Bulgiba. ${ }^{1}$ Faculty of Medicine, University of Malaya, Kuala Lumpur, Malaysia; ${ }^{2} J u l i u s$ Centre University of Malaya, Kuala Lumpur, Malaysia

Introduction Workplace wellness programs are proven to be an important strategy to prevent cardiovascular diseases and stroke. Therefore, a workplace wellness program was conducted among a work cohort in Kuala Lumpur with the aim of improving risk factors for cardiovascular disease and stroke.

Methods This was an open cohort where all employees aged 35 years and above in the workplace were invited to participate in the wellness program. This program involved voluntary yearly screening with results dissemination, lifestyle counselling or referral for medical treatment when necessary. Ethics clearance and informed consent were obtained. Fasting blood glucose, lipid profile, blood pressure, weight, height and waist circumference were measured following standard protocols. This paper will report the findings over a period of 3 years (2008-2010).

Results Majority of this cohort $(\mathrm{n}=1923)$ were Malays $(77.9 \%)$, followed by Chinese (8.4\%), Indians $(9.9 \%)$ and others $(3.8 \%)$. The proportion of female participants was $53 \%$. The mean age of this cohort was $48.5+5.2$ years at baseline. In the General Linear Model after adjusted for race and sex, LDL cholesterol and total cholesterol levels were significantly improved after 3 years. Their mean $(95 \% \mathrm{CI})$ total cholesterol levels over the 3 years $(2008-2010)$ were $5.59(95 \%$ CI 5.49 to 5.69 ), 5.47 (5.40 to 5.55 ) and 5.39 (5.32 to 5.47 ) $\mathrm{mmol} / \mathrm{l}$ respectively. The mean LDL cholesterol levels were 3.59 (3.52 to 3.66), 3.49 (3.43 to 3.26 ) and 3.33 (3.56 to 3.40 ) $\mathrm{mmol} / \mathrm{l}$ respectively. Conclusions Our findings show that low intensity workplace wellness program is effective in improving some cardiovascular risk factors.

\section{SP1-55 WEOAYA: A WHOLE POPULATION CARDIOVASCULAR SCREENING PROGRAMME IN ABU DHABI, UNITED ARAB EMIRATES}

doi:10.1136/jech.2011.142976n.32

C Hajat, ${ }^{*} 0$ Harrison, Z Al Siksek. Department of Public Health \& Research, Health Authority Abu Dhabi, Abu Dhabi, United Arab Emirates

Introduction Data have shown the UAE to have high rates of cardiovascular disease but the risk factor burden remained poorly studied. This study describes the baseline cardiovascular risk profile of the National population of Abu Dhabi.

Methods Adults aged 18 years or over were screened using selfreported indicators, anthropometric measures and blood tests in primary care.

Results The study included 50138 subjects. Mean age (SD) was 36.82 (14.30) years with 21663 (43\%) males and 28474 (57\%) females. Numbers and crude prevalence rates were for obesity 17556 (35\%), overweight 15823 (32\%), central obesity 27480 (55\%), diabetes 8528 (18\%), pre-diabetes 13127 (27\%), dyslipidaemia 21655 (44\%) and hypertension 11377 (23.1\%). Smoking rates were $5570(26 \%)$ in males and $221(0.8 \%)$ in females. Age-standardised rates for diabetes and pre-diabetes were 11792 (25\%) and 14158 (30\%), obesity and overweight were 19711 (41\%) and 16298 (34\%). Family history of premature cardiovascular disease was independently associated with a past history of cardiovascular disease with an OR of 5.34 (95\% CI 3.79 to 7.52 ).

Conclusion This population-wide cardiovascular screening programme in the Middle East has demonstrated a very high cardiovascular burden for this small and young population. The data form a baseline against which progress is monitored for the population-wide Abu Dhabi Cardiovascular Disease Programme.

\section{SP1-56 ASSOCIATION BETWEEN OVERWEIGHT, OBESITY AND SELF-PERCEPTION OF BODY WEIGHT IN ADULTS}

doi:10.1136/jech.2011.142976n.33

${ }^{1} \mathrm{G}$ França, ${ }^{*} \mathrm{D}$ Gigante, ${ }^{2} \mathrm{M}$ T Olinto. ${ }^{1}$ Universidade Federal de Pelotas, Pelotas, Rio Grande do Sul, Brazil; ${ }^{2}$ Universidade do Vale do Rio dos Sinos, Rio Grande do Sul, Brazil

Introduction This study aimed to examine the association between overweight, obesity and self-perception of body weight in adults.

Methods A cross-sectional population-based study was carried out in the city of Pelotas, southern Brazil, with a sample of individuals aged 20-59 years. Weight and height of the participants were measured by previously trained evaluators. Overweight and obesity were defined as body mass index $\geq 25 \mathrm{~kg} / \mathrm{m}^{2}$ and $\geq 30 \mathrm{~kg} / \mathrm{m}^{2}$, respectively. Self-perceived body weight status was directly and indirectly assessed. The participants were first asked whether they perceive themselves as too thin, thin, normal, fat or too fat and then the difference between reported ideal and actual body weight measured after the interview was calculated. 
Results A total of 1894 adults participated in the study, of which $57.4 \%$ were women. The prevalences of overweight and obesity were $58 \%$ and $23.8 \%$, respectively. About $40 \%$ of the participants reported they perceived their weight as normal. However, when selfperceived body weight was indirectly assessed, $63.3 \%$ reported a desire to weigh less. Among the overweight and obese participants, $61 \%$ and $86.4 \%$ perceived themselves as fat or very fat, respectively, and reported a desire to weigh less.

Conclusion In this sample with high prevalences of overweight and obesity, most participants perceived their excess weight and reported a desire to weigh less. This seems an ideal setting for the implementation of interventions targeting weight loss and promoting healthy habits.

\section{SP1-57 EUROPEAN BONE TUMOUR OUTCOME STUDY (EBTOS): OVERVIEW OF HEALTH RELATED OUALITY OF LIFE (OOL) IN STUDY COHORT}

doi:10.1136/jech.2011.142976n.34

${ }^{1} \mathrm{M}$ Brown, ${ }^{1} \mathrm{M}$ Pearce, ${ }^{*}{ }^{1} \mathrm{~J}$ Salotti, ${ }^{2} \mathrm{~A}$ Craft, ${ }^{3} \mathrm{~J}$ Hale. ${ }^{1}$ Institute of Health \& Society, Newcastle University, Newcastle Upon Tyne, UK; ${ }^{2}$ Sir James Spence Institute, Royal Victoria Infirmary, Newcastle Upon Tyne, UK; ${ }^{3}$ Paediatric Oncology, Royal Victoria Infirmary, Newcastle Upon Tyne, UK

Introduction Survivors of bone tumours diagnosed in childhood or early adulthood are reported to have more impairment of health related QOL than most other cancer survivor groups. Reports of the relationship between QOL and local treatment strategy have been inconsistent. To clarify this issue a European cohort of bone tumour survivors was identified. This report forms a baseline for further analyses.

Methods Osteosarcoma and Ewing's sarcoma survivors were $>5$ years from diagnosis, $<40$ yrs at diagnosis and $>16$ yrs at time of survey. 1145 eligible survivors were mailed a questionnaire which included socio-demographic data, recall of treatment, SF36, Rosenberg Self Esteem Scale, EORTC Body Image \& Sexuality modules and TESS to assess physical function. Statistical analysis was with $\chi^{2}$ and t test using Stata software.

Results 714 (62\%) survivors returned questionnaires (253 UK, 31 Netherlands, 430 DE). Median age at diagnosis was 15 yrs (1-38) and at survey was $26 y$ ys (16-52). Time since diagnosis was 5-20 yrs (median 11). Survivors had: Ewing's sarcoma (319), osteosarcoma (395), upper extremity site (107), lower (535), axial (72), amputation (161), rotationplasty (51), limb salvage (502). Most were single and childless, only $6 \%$ were unemployed while $64 \%$ felt that job opportunities were affected. SF36 scores showed impaired physical (UK and DE) and mental health (DE) compared to population norms, but enhanced self esteem. There were national differences in sub-scale scores.

Conclusions This overview of the EBTOS cohort confirms areas of impaired QOL balanced by enhanced self-esteem, while also demonstrating variability between the national groups.

\section{SP1-58 MALNUTRITION, QUALITY OF LIFE AND CANCER: ASSOCIATION BETWEEN DIFFERENT NUTRITIONAL PARAMETERS}

doi:10.1136/jech.2011.142976n.35

${ }^{1} \mathrm{~S}$ P Orlandi, ${ }^{1} \mathrm{M}$ C F Assunção, ${ }^{2} \mathrm{C}$ A Pastore, ${ }^{2} \mathrm{M}$ C Gonzalez. ${ }^{1}$ Post Graduation in Epidemiology, Federal University of Pelotas, Pelotas, Rio Grande do Sul, Brazil, ${ }^{2}$ Post Graduation in Health and Behaviour, Catholic University of Pelotas, Pelotas, Rio Grande do Sul, Brazil

Introduction Weight loss is a frequent complication in patients with cancer, and it is present in almost $85 \%$ of patients in some specific kinds of tumour. Malnutrition, decreased functional capacity and quality of life (QOL) contribute to an increased morbidity and mortality in these patients.

Objectives To evaluate the association among nutritional status, quality of life, index of fat free mass (FFMI) and functional capacity in patients undergoing chemotherapy.

Methods A prospective study was conducted in patients before the first chemotherapy course in the Hospital of Federal University of Pelotas, Brazil. Nutritional status was determined by PatientGenerated Subjective Global Assessment and quality of life by the European Organization for Research and Treatment of Cancer Quality of Life Questionnaire. Bioelectrical impedance analysis and hand grip strength (HGS) were performed to evaluate FFMI and functional capacity, respectively.

Results 75 patients were evaluated, $74.7 \%$ had cancer of the digestive system. Only $12.8 \%$ of the patients were classified as well nourished. HGS median was $26 \mathrm{~kg}$ (IOR:18; $32 \mathrm{~kg}$ ) FFMI median was $17.5 \mathrm{~kg} / \mathrm{m}^{2}$ (IOR:16.1; $18.8 \mathrm{~kg} / \mathrm{m}^{2}$ ). Severely malnourished patients had a worse functional capacity $(p=0.01)$; lower FFMI $(p=0.005)$ and lower general QOF scores $(p=0.03)$ than the nourished ones. A significant positive correlation was found between IFFM and HGS $(r=0.51 ; p<0.001)$ and significant negative correlations between functional capacity and general $\mathrm{OOL}(\mathrm{r}=-0.48$; $\mathrm{p}<0.001)$ and HGS $(\mathrm{r}=-0.44 ; \mathrm{p}<0.001)$.

Conclusions Malnutrition is a determining factor in the prognosis and QOL in these patients and it can be assessed by different parameters such as body composition, subjective evaluations or changes in functional capacity.

\section{SP1-59 DEPRESSION AMONG ELDERLY PEOPLE IN AN OLD HOME OF DHAKA CITY}

doi:10.1136/jech.2011.142976n.36

${ }^{1} \mathrm{M}$ Moula, ${ }^{1} \mathrm{~S}$ J Mumu, * ${ }^{2} \mathrm{M}$ S Flora. ${ }^{1}$ Bangladesh Institute of Health Sciences (BIHS), Dhaka, Bangladesh; ${ }^{2}$ National Institute of Preventive and Social Medicine (NIPSOM), Dhaka, Bangladesh

Introduction With a rapidly ageing society, geriatric depression is emerging as important public health concern. The untreated depressed elderly patients have significant clinical and social implications as these disorders decrease an individual's quality of life and increase dependence on others. The present study aimed to assess the level of depression and its associated factors among the elderly (60 years old and above) in an old home.

Methods Under a cross sectional design 107 respondents aged $>60$ years were selected purposively from an Old Home of Dhaka city, Bangladesh. A 30-item Geriatric Depression Scale (GDS) questionnaire was used to assess the level of depression with a score $\leq 9$ as normal or non-depressive, $10-19$ as mild and 20-30 as severely depressed.

Results Mean age of study participants was $69.4 \pm 5.1$ years and $63 \%$ were male, $46.7 \%$ illiterate, $61.7 \%$ from rural area. About half of elderly respondents were having some degree of depression of whom $8.4 \%$ were severely depressed. Depression was more common in females $(60 \%)$ than males $(40 \%)$. Depression was significantly associated with nuclear family $(p=0.009)$, low family income $(p=0.001)$, female gender $(p=0.048)$, chronic illness $(p=0.042)$ and widow or widowers $(p=0.036)$. No influence of educational status and living area was found.

Conclusion This study showed that proportion of depression was high among elderly and depression was significantly associated with nuclear family, female gender, chronic illness, widow/ widower and low family income. Along with other illness depression should also be one of the key areas of concern for the elderly population. 